Rev. Elev, Méd. vét. Pays trop., 1974, 27 (1) : 61-65

\title{
Mycobacterium farcinogenes, agent causal du farcin du bouf en Afrique (*)
}

\author{
par G. CHAMOISEAU (**)
}

\begin{abstract}
RESUME
En Afrique, le farcin du bceuf est dû à une mycobactérie atypique et non à Nocardia farcinica. Les souches de cette mycobactérie se partagent en deux biotypes qu'il n'est pas possible d'assimiler à $N$. farcinica Trevisan 1889. Mycobacterium farcinogenes var. senegalense et var. tchadense est proposé pour désigner désormais l'agent causal du farcin du bcuf en Afrique. Cette espèce (et ses deux variétés) présente des caractères qui lui sont particuliers et elle se classe, en raison des acides mycoliques qu'elle renferme, dans le genre Mycobacterium.
\end{abstract}

\section{INTRODUCTION}

Le farcin est une maladie infectieuse des bovins qui sévit communément dans certains pays d'Afrique de l'Ouest (14), du Centre (20, $21)$ et de l'Est $(2,15,16,17,18)$. Il est appelé actinomycose lymphatique, nocardiose bovine, farcin du bouf, par les auteurs de langue française; il est appelé \& lymphangitis of cattle», «bovine nocardiosis », *bovine farcy * et "cattle farcy par les auteurs de langue anglaise. Le farcin se caractérise, en général, par une inflammation suppurative des vaisseaux et ganglions lymphatiques superficiels, avec formation de cordes, de nodules et d'adénopathies. Parfois, l'infection se généralise et des pseudotubercules apparaissent sur les différents viscères, les grandes séreuses et dans les ganglions lymphatiques profonds.

Les descriptions de la maladie au Sénégal (14), au Tchad $(20,21)$, au Soudan $(2,15,16,17$,

(*) Paru in: Ann. Microbiol. Inst. Pasteur, 1973, 124 A : 215-222. Reproduit avec l'aimable autorisation du Directeur de ce périodique le Docteur P. MEYER et des Editeurs Masson et Cie.

(**) I.E.M.V.T., 10, rue Pierre Curie, 94700 Maisons-Alfort (France). Adresse actuelle: B.P. 175, Nouakchott (Mauritanie).
18), font régulièrement état de manifestations cliniques, d'aspects anatomo-pathologiques et de réactions immunologiques identiques à des variantes mineures près. Par ailleurs, en certains points, ces descriptions rappellent fort celle que, en 1888, Nocard donnait du farcin du bœuf en Guadeloupe (19).

Il est alors compréhensible que l'agent responsable du syndrome africain, ce micro-organisme filamenteux et ramifié, ait été assimilé à Nocardia farcinica Trevisan 1889, responsable du syndrome guadeloupéen. Si l'apparence microscopique du germe explique cette extrapolation, des observations d'ordre bactériologique récentes vont à l'encontre de cette assimilation à une Nocardia et singulièrement à l'espèce-type $N$. farcinica asteroides $(10,11)$. De plus une série de travaux $(1,6,7,12,20)$ a permis d'établir que l'actinomycète africain n'est pas une Nocardia, mais une mycobactérie authentique, que sa composition lipidique particulière autorise même à classer parmi les mycobactéries dites « atypiques > $(1,12)$.

Le but du présent travail est, en résumant nos travaux $(1,6,7,12,22)$, de présenter cet actinomycète, de préciser sa position taxonomique et de proposer, pour le désigner, une 
appellation tenant compte de sa nature véritable et de ses particularités.

\section{MATERIEL ET METHODES}

Toutes les souches considérées au cours de nos travaux ont été régulièrement isolées de lésions suppurées des parenchymes et des ganglions de bovins d'Afrique centrale (Tchad) et d'Afrique occidentale (Sénégal) atteints de farcin.

Elles ont été étudiées comparativement du point de vue de leur morphologie à l'état frais et en culture $(6,22)$, de leur comportement biochimique à l'égard de certains substrats (6, 7,22 ), de leur composition lipidique après extraction des lipides par l'alcool-éther et le chloroforme, et de leur analyse par chromatographie sur couche mince et en phase gazeuse $(1,12)$. Nous avons aussi étudié leur pouvoir pathogène expérimental pour le cobaye (22).

\section{RESULTATS}

Nous pensons qu'il est possible d'étendre aux souches isolées au Soudan les résultats des observations faites sur les souches isolées au Tchad, car elles ont de nombreux traits communs.

\section{A. Morphologie}

1. Etat frais. - Dans le pus, le germe se présente - après coloration par la méthode de Ziehl ou celle de Gram - selon l'image classique de filaments plus ou moins longs, flexueux, porteurs de ramifications d'importance variable.

Ces filaments sont, en général, rassemblés en touffes, formant un lacis inextricable. Dans le pus des lésions closes, il est pratiquement en culture pure, ce qui facilite considérablement son isolement. Dans les coupes histologiques d'organe malade, l'actinomycète se présente sous la même apparence microscopique $(6,18$, 20).

2. En culture. - Sur les milieux de Coletsos, de Loewenstein-Jensen, ou sur la gélose tryptose-sérum de cheval, les colonies sont « rough », surélevées, montrant au début une architecture régulière, faite d'éléments sphériques, et prenant par la suite des formes cérébroïdes $(6,18,20,22)$. Les souches tchadiennes isolées donnent après 10 à 20 jours d'incubation à $37^{\circ} \mathrm{C}$ des colonies pigmentées en jaune miel, sèches, dures et dont les genres s'émulsionnent très difficilement dans l'eau distillée. Les souches sénégalaises par contre, se présentent, en 24 à 48 heures, sous la forme de colonies pigmentées en jaune ocre, moins dures, moins sèches, s'émulsionnant plus facilement. Sur les milieux opaques, à l'œuf, on peut voir tout autour des grosses colonies ou des groupes de colonies - de l'un ou l'autre type de souche - une auréole irisée due, semble-t-il, à la sécrétion par les germes d'une substance grasse qui s'étalerait par vagues $(6,21)$.

En milieu liquide, bouillon tryptose-sérum par exemple, la culture se fait en voile rugueux, sec, blanchâtre, flottant sur un milieu restant clair $(6,18,20,22)$. Souches tchadiennes et sénégalaises gardent, là aussi, leur vitesse respective de croissance. Il faut noter que, sur milieu de Sauton, les souches tchadiennes, à l'inverse des souches sénégalaises, ne se développent pratiquement pas $(6,20)$.

\section{B. Comportement biochimique}

Les deux types de souches ont en commun une catalase puissante et une nitrate réductase; elles ne produisent pas d'acide nicotinique, ne transforment pas le citrate de fer ammoniacal et ne fixent pas le rouge neutre $(6,22)$.

Leur activité amidasique présente de légères variations seulement sur le plan quantitatif (7) : les souches sénégalaises dites "rapides 》 ont une activité puissante à l'égard de l'acétamide, la formamide, l'allantoïne, la carbamide, la benzamide, l'isonicotinamide, la malonamide, la nocotinamide (7); les souches tchadiennes dites «lentes», par contre, n'attaquent fortement que l'acétamide et la formamide et sont moins actives sur les autres amides (7). Les souches des deux types n'ont à l'égard de la salicylamide qu'une activité réduite (7).

Les divergences sont plus nettes quant à leur comportement à l'égard des sucres, des acides organiques, des substances azotées. Les souches tchadiennes attaquent le glucose et le lévulose; elles utilisent le pyruvate, le citrate et le benzoate de sodium; elles ont un pouvoir protéolytique quasi nul. Les souches sénégalaises attaquent, en plus du glucose et du lévulose, le saccharose, la mannite, le glycérol; elles utilisent 
le malonate de sodium et, de plus, sont particulièrement actives sur l'urée $(20,22)$.

Enfin, les deux types de souches sont parfaitement résistants à l'INH et au PAS $(6,22)$.

\section{Composition lipidique}

La présence de l'auréole irisée autour des colonies a particulièrement retenu notre attention. L'analyse des lipides a révélé chez les deux types de souches - en plus des composants normaux des bactéries de l'ordre des Actinomycétales - la présence d'acides gras à chaîne normale supérieure à 20 carbones dans les acides gras totaux. On y constate aussi la présence d'acides gras $\beta$-hydroxylés du type mycolique, pyrolysables avec libération d'acide tétracosanoïque, ce qui caractérise la nature de mycobactérie atypique des souches (1). Il faut noter la présence d'un «cord factor * d'un type inconnu chez les Nocardiae et l'absence d'acide nocardomycolique (1).

Chez les souches sénégalaises, la présence d'un mycoside C', forme simplifiée du mycoside $\mathrm{C}$ (cette dernière substance caractérisée seulement chez les mycobactéries atypiques) est une particularité rare $(1,12,22)$.

\section{Pouvoir pathogène expérimental}

Chez le cobaye, des injections sous-cutanées de cultures des deux types de souches, obtenues en bouillon nutritif, provoquent la formation d'abcès qui fistulisent au bout d'un temps plus ou moins long et guérissent lentement $(20,22)$.

L'injection intrapéritonéale révèle le tropisme particulier des germes pour la sphère génitale, avec formation régulière d'orchites, d'abcès intratesticulaires, d'abcès de la vaginale et des vésicules sếminales. Les abcès du péritoine viscéral et pariétal sont importants, mais rares quand il s'agit des souches tchadiennes, miliaires et très nombreux quand il s'agit des souches sénégalaises. Il n'y a jamais hypertrophie de la rate $(2,22)$; il y a toujours sensibilisation aux tuberculines « humano-bovine * et aviaire $(2,20,22)$. La mort termine toujours l'évolution de la maladie expérimentale (22). Enfin, le germe est régulièrement retrouvé en culture pure faite à partir du pus des lésions avec toutes les caractéristiques morphologiques décrites plus haut (22).

De cet ensemble de faits d'où émerge, avec un relief tout particulier, ce qui a trait à la composition lipidique $(1,12)$, il faut conclure que le farcin des bovins en Afrique n'est pas dû à une Nocardia, mais bien à une authentique mycobactếrie. Les particularités de cette mycobactérie, agent causal du même syndrome, font conclure à l'existence de deux variétés du même germe.

\section{DISCUSSION}

La nécessité de réviser la position taxonomique de cet actinomycète responsable des farcins africains s'impose. Elle passe donc des Nocardiaceae, où l'avait rangée une simple extrapolation, aux Mycobacteriaceae.

En dépit des apparences, il ne s'agit pas, pour nous, de réviser du même coup la classification de l'espèce Nocardia farcinica Trevisan 1889. Le remarquable travail de LECHEVALIER, HORAN, LECHEVALIER (13), l'observation de CHAMOISEAU et ASSELINEAU (8), démontrent bien que des souches de $N$. farcinica de différentes collections sont, elles aussi, des mycobactéries, et incitent à se demander si $N$. farcinica a jamais existé en tant que Nocardia. On peut même se demander comme NEUMANN (in 16) si le bœuf sénégalais, en introduisant en Guadeloupe la tique sénégalaise, n'y a pas du même coup introduit l'actuelle mycobactérie atypique sénégalaise. Du pus et des prélèvements qu'envoya COUZIN (16) de la Guadeloupe, NOCARD isolait, en effet, un actinomycète à Gram positif prenant la coloration de Ziehl et se présentant en touffes comme dans nos lésions africaines actuelles et non en filaments isolés ou en petits grains comme dans des lésions à $N$. asteroides ou $N$. brasiliensis. Mais ce ne sont là que de vaines hypothèses. La description très vague qu'a laissée NOCARD (19) de sa souche et le fait que cette dernière a disparu de toutes les collections (13) sans espoir de la réisoler ou d'en étudier une autre puisque le farcin a disparu des Antilles françaises (information des Services vétérinaires) enlèvent toutes possibilités de vérifier si $N$. farcinica Trevisan 1889 (3) doit être considérée désormais comme une mycobactérie plutôt que comme une Nocardia. Ce dernier point, jusqu'à ce qu'une démonstration plus rigoureuse soit faite, reste à élucider.

S'il ne peut être question de déclasser et de rebaptiser $N$. farcinica Trevisan 1889, il s'avère 
nécessaire, par contre, de faire une description la plus précise possible de l'actinomycétale africaine. Celle-ci doit prendre son rang parmi les mycobactéries dites \& atypiques » du fait de sa composition lipidique et de certaines propriétés biochimiques (résistance à certains antibiotiques, activité amidasique, par exemple) (1, $4,5,7,12,23)$. Dans ce groupe, elle se singularise cependant par sa morphologie typique - - et surtout particulièrement stable dans les lésions et en culture (sa provenance et son apparence microscopique suffiraient presque à assurer le diagnostic), par une activité malonamidasique que l'on ne rencontre jamais chez les autres mycobactéries atypiques décrites $(4,5,23)$ et par un pouvoir pathogène particulièrement net et régulier pour le cobaye, ce qui est exceptionnel pour une mycobactérie atypique $(4,5,23)$.

C'est pourquoi nous croyons pouvoir - conformément aux prescriptions du Code International de Nomenclature des Bactéries (7) proposer pour la désigner le binôme de $\mathrm{Myco}$ bacterium farcinogenes:

- Mycobacterium, nom de genre, en raison spécialement de la nature des composants lipidiques du germe (principe 10, chapitre $2 \mathrm{du}$ Code);

- Farcinogenes, nom d'espèce (règle 6, chapitre 3 du Code), signifie " qui engendre le ou un syndrome farcin» identique quant à la clinique et à l'anatomie pathologique à celui qu'on trouve à l'ouest, au centre, à l'est de l'Afrique. Le syndrome présenté par un farcin guadeloupéen pourrait être éventuellement une entité différente.
Pour tenir compte des variations présentées par les souches selon leur biotype, nous proposons les noms des sous-espèces (règle 7 , chapitre 3 du Code) :

- Senegalense, pour les souches sénégalaises ou de type sénégalais, à croissance rapide et activité amidasique intense;

- Tchadense, pour les souches tchadiennes ou de type tchadien, à croissance lente et à activité amidasique réduite.

Si bien que l'actinomycétale du farcin africain se classerait désormais de la façon suivante :

- Ordre des Actinomycetales;

- Famille des Mycobacteriaceae;

- Genre Mycobacterium;

- Espèce farcinogenes; var. senegalense et var. tchadense.

\section{Remerciements}

Nos remerciements vont à Monsieur le Professeur J. Asselineau ainsi qu'à ses collaborateurs G. et M. A. Laneelle de la Faculté des Sciences de Toulouse, pour leur collaboration décisive, à Monsieur le Professeur $\mathrm{H}$. et à Mme M. P. Lechevalier, à Monsieur 1e Professeur Waksmann de Rutgers University (New Jersey, USA), à mes confrères A. Provost du Laboratoire de Farcha, P. Perreau de l'I.E.M.V.T. (Maisons-Alfort), M. Vigier de l'I.V.I. (Debré-Zeit, Ethiopie) qui ont facilité mes travaux et à Messieurs les Vétérinaires Inspecteurs généraux J. Pagot et $M$. Thomé de l'I.E.M.V.T.

\section{SUMMARY}

Mycobacterium farcinogenes, a causal agent of bovine farcy in Africa

In western, central and eastern Africa, bovine farcy is rife with the same clinico-pathological and immunological features. The causal agent is an actinomycete. On first relation, this actinomycete was believed to be Nocardia farcinica on account of its microscopical likeness with the causal agent of bovine farcy in the West Indies. Careful studies showed that the african actinomycete is a mycobacterium and isolates from eastern and central Africa are somewhat distinct from those obtained in the west of the continent. It is not possible to assimilate these organisms to Nocardia farcinica Trevisan 1889 . For these reasons the causal agents of bovine farcy in Africa are named: Mycobacterium farcinogenes, var. senegalense and $M$. farcinogenes var. tchadense. 


\section{RESUMEN}

\section{Mycobacterium farcinogenes, agente causal del muermo del buey en Africa}

En Africa, una micobacteria atipica es causa del muermo del buey y no Nocardia farcinica. Las cepas de dicha micobacteria se dividen en dos biotopos que no es posible de asemejar a $N$. farcinica Trevisan 1889. Se propone Mycobacterium farcinogenes var. senegalense y var. tchadense para designar en adelante el agente causal del muermo del buey en Africa. Esta especie ( $y$ sus dos variedades) presenta caracteristicas que son peculiares a ella y se clasifica, a causa de los acidos micolicos que contiene, en el género Mycobacterium.

\section{BIBLIOGRAPHIE}

1. ASSELINEAU (J.), LANEELLE (M. A.) et CHAMOISEAU (G.). De l'étiologie du farcin de zébus tchadiens: nocardiose ou mycobactériose? II. Composition lipidique. Rev. Elev. Méd. vét. Pays trop., 1969, 22 : 205-209.

2. AWAD (F.I.). The interrelationship between tuberculosis and bovine farcy. J. comp. Path., 1958, 68 : 324-330.

3. Bergey's manual of determinative bacteriology $7 \mathrm{e}$ éd. $1957,758 \mathrm{p}$.

4. BUTTIAUX (R.), BEERENS (H.) et TACQUET (A.). Mycobactéries atypiques, in: Manuel de techniques bactériologiques. Paris, Flammarion, 1963 , p. 468.

5. CANETTI (G.) et GROSSET (J.). Mycobactéries dites \& atypiques * in: Techniques et indications des examens bactériologiques en tuberculose. St-Mandé, Editions de la Tourelle (Coll. Techniques de bases).

6. CHAMOISEAU (G.). De l'étiologie du farcin de zébus tchadiens : nocardiose ou mycobactériose? I. Etude bactériologique et bjochimique. Rev. Elev. Méd. vét. Pays trop., 1969, 22 : 195-204.

7. CHamoiseau (G.). De l'étiologie du farcin de zébus tchadiens : nocardiose ou mycobactériose? III. Activité amidasique. Rev. Elev. Méd, vét. Pays trop., 1972, 25 : 191-194.

8. CHAMOISEAU (G.) et ASSELINEAU (J.), Examen des lipides d'une souche de Nocardia farcinica. Présence d'acides mycoliques. C.R. Acad. Sci., Paris, 1970, 270 sér., D : 2603-2604.

9. Comité d'Edition de la Commission de Jugement du Comité international de Nomenclature des Bactéries. Congrès international de Microbiologie, Moscou, 1966, in : Ann. Inst. Pasteur, 1967, 113 : 267-308,

10. GORDON (R. E.). Some strains in search of a genus: Corynebacterium, Mycobacterium, Nocardia or what? J. gen. Microbiol., 1966, 43 : 329-343.

11. GORDON (R. E.), et MIHM (J. M.). A comparative study of some strains received as Nocardiae. J. Bact., 1957, 73 : 15-27.
12. LANEELLE (G.), ASSELINEAU (J.) et CHAMOISEAU (G.). Présence de mycosides $C^{\prime}$ (formes simplifiées de mycoside $C$ ) dans les bactéries isolées de bovins atteints du farcin. FEBS Letters, 1971, 19 : 109-111.

13. LECHEVALIER (M. P.), HORAN (A, C.) et LECHEVALIER (H.). Lipid composition in the classification of Nocardiae and mycobacteria. J. Bact., 1971, 105: 313-318.

14. MEMERY (G.), MORNET (P.) et CAMARA (A.). Premiers cas authentiques de farcin du bouf en Afrique Occidentale Française. Rev. Elev. Méd. vét. Pays trop., 1958, 11: 11-16.

15. MOSTAFA (I. E.). Studies on cattle nocardiosis "Bovine farcy" in the Sudan. Sudan J. vet. Sci. anim. Husb., 1962, 7 : 1-9.

16. MOSTAFA (I. E.). Bovine nocardiosis (cattle farcy). A review. Vet. Bull., 1966, 36: 189-193.

17. MOSTAFA (I. E.). Studies on bovine farcy in the Sudan. I. Pathology of the disease. J. comp. Path., 1967, 77 : 223-229.

18. MOSTAFA (I. E.). Studies on bovine farcy in the Sudan. II. Mycology of the disease. J. comp. Path., 1967, 77: 231-237.

19. NOCARD (E.). Note sur la maladie des boufs de la Guadeloupe connue sous le nom de farcin. Ann. Inst. Pasteur, 1888, 2 : 297-302.

20. PERPEZAT (A.), DESTOMBES (P.) et MARIAT (F.). Etude histopathologique de la nocardiose du bouf au Tchad et caractères biochimiques de Nocardia farcinica. Rev. Elev. Méd. vét. Pays trop., 1967, $20: 429-435$.

21. PERPEZAT (A.), MARIAT (F.), DESTOMBES (P.) et THOME (M.). Importance du farcin chez le zébu du Tchad. Bull. Soc. Path. exot., 1963, 56: 375-383.

22. Rapports annuels du Laboratoire de Recherches vétérinaires de Farcha, Fort-Lamy, Tchad, 1969. 70-71.

23. RULLIER (J.) et PARODI (A.). Mycobacterium in: Laboratoire et diagnostic en médecine vétérinaire. Paris, Vigot Frères, 1968 , p. 465 et sujvantes. 